\title{
Wide-Band Electromagnetic Scattering from a Dielectric BOR Buried in a Layered Lossy Dispersive Medium
}

\author{
Norbert Geng, Member, IEEE, and Lawrence Carin, Senior Member, IEEE
}

\begin{abstract}
A method of moments (MoM) analysis is developed for electromagnetic scattering from a dielectric body of revolution (BOR) embedded in a layered medium (the half-space problem constituting a special case). The layered-medium parameters can be lossy and dispersive, of interest for simulating soil. To make such an analysis tractable for the wide-band (short-pulse) applications of interest here, we have employed the method of complex images to evaluate the Sommerfeld integrals characteristic of the dyadic layered-medium Green's function. Example wideband scattering results are presented, wherein fundamental wave phenomenology is elucidated. Of particular interest, we consider wide-band scattering from a model plastic mine, buried in soil, with the soil covered by a layer of snow.
\end{abstract}

Index Terms - Method of moments, subserfice sensing, timedomain scattering.

\section{INTRODUCTION}

$\mathbf{E}$ LECTROMAGNETIC scattering from bodies of revolution has been a subject of interest for more than three decades [1]-[9]. This research has been motivated by the fact that a body of revolution (BOR) can be used to simulate many man-made targets (e.g., missiles). Moreover, while a BOR is three-dimensional (3-D), the scattering of waves from such a target can be solved by using what is often a 2.5 -dimensional analysis, wherein the target's azimuthal symmetry is exploited. In particular, azimuthal Fourier modes are used to represent the target's rotational variation, thereby reducing the problem to finding the unknown fields or currents along a two-dimensional (2-D) "generating arc" [1]-[9]. Such unknowns are usually solved for in the frequency domain using a method of moments (MoM) analysis [1]-[9].

Most previous such research has focused on targets in free space, representative of scattering from airborne BOR's. In such work one exploits the free-space Green's function, which can be computed in closed form and is itself rotationally symmetric; these two properties are of particular importance in simplifying the MoM numerical analysis. However, there has recently been significant interest in using electromagnetic scattering (radar) for the detection of underground targets

Manuscript received January 13, 1998; revised Januarh 28, 1999.

The authors are with Department of Electrical and Computer Engineering, Duke University, Durham, NC 27708-0291 USA.

Publisher Item Identifier S 0018-926X(99)04766-3.
[10]-[14]. Examples of such include buried mines, unexploded ordnance, and buried pipes [13], [14]. Each of these manmade targets has particular properties that can be exploited in the context of the scattering analysis. In the work presented here, we are interested in buried mines. It is well known that such targets often closely resemble BOR's [15]. This property has been exploited in a recent series of papers [16]-[19], in which MoM was used to analyze wide-band scattering from buried conducting BOR's, which simulated metallic mines.

The previous research on buried metal mines demonstrated the close agreement that can be obtained between theory and experiment [19], for real mines, and shed light on the underlying wave phenomenology. However, a principal challenge in mine detection involves plastic mines, with such targets generally composed of only trace metallic content (usually the tiny firing pin, often representing only a few grams of metal). In the work presented here, we therefore consider dielectric BOR's buried in a layered medium, with the lossy, dispersive layers representing the typical layered character of many soils (and/or a snow layer).

While scattering from a plastic mine can be solved via a 3-D finite-difference time-domain (FDTD) algorithm, such requires solution of the fields at all points in the computational domain [20]. Though ceding some generality, the MoM formulation only requires solution of the fields along the BOR generating arc, from which the scattered fields can be determined at any point. Moreover, a modified version of the BOR-MoM code can be used to study the properties of dielectric-mine resonances [21], something that is difficult to perform via FDTD, since mines are generally characterized by low- $Q$ resonances. Finally, the exploitation of the mine's symmetry yields important phenomenological insight. In particular, for the case of buried-BOR targets, one can readily demonstrate [22] that there are no cross-polarized fields $(H V=V H=0)$ in the case of backscattering, a property that can be exploited for target discrimination.

The analysis presented here is not the first to model buried dielectric discontinuities as BOR's. Chang and Mei [23] used a hybrid algorithm that combined finite-element method (FEM) modeling of the BOR with an integral equation, the latter accounting for the surrounding half-space medium. Although 
that work did not consider buried mines (it focused on geological discontinuities), the general framework could be applied to the case of dielectric mines. However, the FEM requires solution of the fields at all points within the BOR, while, again, the MoM analysis only requires solution for the fields on the surface of the generating arc. This of course results in some loss of generality, but yields significant computational savings, which are beneficial for the wide-band (short-pulse) applications of interest.

The BOR-MoM analysis developed here uses the mixedpotential integral-equation formulation of Michalski and Zheng [24] (what they termed "formulation C"). To effect such, one requires the space-domain dyadic Green's function for a general layered medium, here considering loss and dispersion as well. As is well known, closed-form representation of the dyadic Green's function components is only possible in the spectral domain [24], while the Sommerfeld integrals required for conversion to the space domain must be evaluated approximately [25]-[29]. Over the years various numerical and asymptotic techniques have been developed for evaluating these ubiquitous integrals [25]-[29]. Most electromagnetic mine-detection systems are of an ultra-wide-band nature [11], [14], [19] and, therefore, it is essential that the Green's function components (which are frequency dependent) be computed as efficiently as possible. To this end, we exploit here the method of complex images [27]-[29].

The method of complex images utilizes a parametric algorithm, for example Prony's method [30], [31] or the matrixpencil method [32], [33], to express the components of the spectral-domain dyadic Green's function in terms of a sum of exponentials with complex arguments (a different such representation for each component of the dyadic). Utilizing the Weyl or Sommerfeld identity [27]-[29], each term in the summation can then be converted to the space domain (in closed form), interpreted physically as an image in complex space. Therefore, the problem of determining the space-domain Green's function for a layered medium is reduced from the laborious and computationally expensive task of evaluating Sommerfeld integrals numerically [34], to the relatively efficient task of parametric estimation [30]-[33]. As noted, the method of complex images has been used previously, primarily for conducting targets or radiators [16]-[19], [27]-[29], [35], while here we are interested in dielectric targets, thereby necessitating additional Green's function components [36].

In addition to describing the above formulation, with a focus on computational efficiency for wide-band applications, we examine the phenomenology associated with scattering from buried dielectric and conducting targets. Of particular interest are the effects of target and soil properties on the subsequent target signature, as a function of operating frequency. Moreover, the use of a layered-media Green's function, rather than the simplified half-space Green's function [16]-[19], allows us to examine several important and realistic scattering problems of interest. For example, one may be interested in detecting small mines buried in soil, with the soil covered by a snow layer. This is a problem well suited to the numerical paradigm

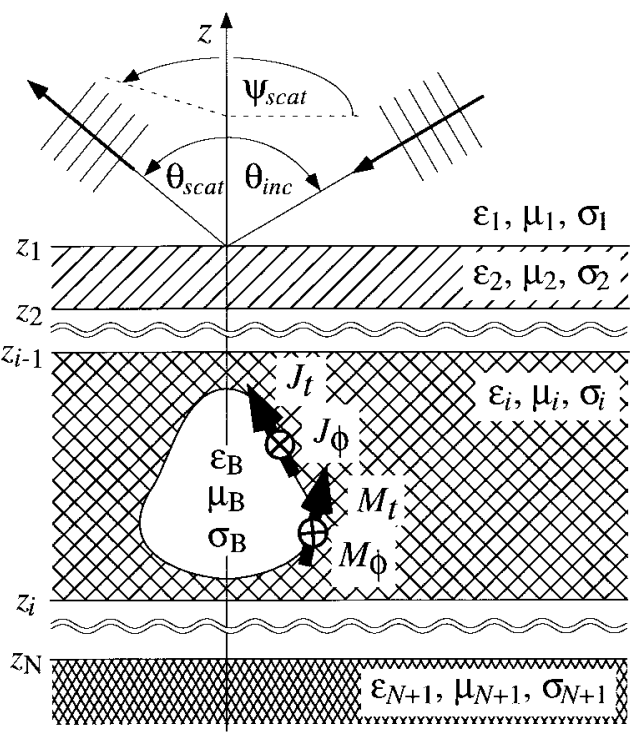

Fig. 1. Plane-wave scattering from body of revolution in planar multilayered environment.

utilized here, and the phenomenology of such is examined in detail, as a function of snow type (wetness) and snow depth.

The remainder of the text is organized as follows. The numerical formulation is discussed in Section II, wherein we detail the general MoM formulation and use of complex images. In Section III are presented several wide-band (short-pulse time-domain) numerical results, wherein basic phenomenology is examined for realistic model parameters (including lossy and dispersive soil and snow). Conclusions are addressed in Section IV.

\section{THEORY}

We consider scattering from a (lossy) dielectric BOR situated in a planar layered environment, assuming the BOR's axis of rotation is normal to the interfaces (Fig. 1). We are interested in the scattered time domain fields for an incident ultrawideband short-pulse plane wave. The problem is analyzed in the frequency domain using a MoM solution of the coupled surface integral equations, with the time-domain response calculated via a fast Fourier transform (FFT). For the wideband problem of interest here, the frequency-domain analysis must be as efficient as possible. Therefore, the dyadic and scalar spatial domain Green's functions are calculated using the complex image technique introduced in [27]-[29], avoiding numerical integration of Sommerfeld type integrals.

\section{A. Surface Integral Equation Formulation}

By enforcing boundary conditions for the tangential electric and magnetic field components on the BOR surface, one obtains coupled integral equations for the electric and magnetic surface currents, $\boldsymbol{J}$ and $\boldsymbol{M}$, respectively. These integral equations can be put in a mixed potential form (MPIE, mixed 
potential integral equation)

$$
\begin{aligned}
& \boldsymbol{n} \times \boldsymbol{E}^{\mathrm{inc}}(\boldsymbol{r})=\boldsymbol{n} \times\left[j \omega \mu(\boldsymbol{r}) \int_{S} \stackrel{\overrightarrow{\boldsymbol{K}}}{A}_{A}\left(\boldsymbol{r}, \boldsymbol{r}^{\prime}\right) \cdot \boldsymbol{J}\left(\boldsymbol{r}^{\prime}\right) d S^{\prime}\right. \\
& -\frac{\nabla}{j \omega \varepsilon(\boldsymbol{r})} \int_{S} K_{\phi e}\left(\boldsymbol{r}, \boldsymbol{r}^{\prime}\right) \nabla \cdot \boldsymbol{J}\left(\boldsymbol{r}^{\prime}\right) d S^{\prime} \\
& \left.+\nabla \times \int_{S} \stackrel{\leftrightarrow}{\boldsymbol{G}}_{F}\left(\boldsymbol{r}, \boldsymbol{r}^{\prime}\right) \cdot \boldsymbol{M}\left(\boldsymbol{r}^{\prime}\right) d S^{\prime}\right]_{\boldsymbol{r} \in S^{+}} \\
& +\boldsymbol{n} \times\left[j \omega \mu_{B} \int_{S} G_{B}\left(\boldsymbol{r}, \boldsymbol{r}^{\prime}\right) \boldsymbol{J}\left(\boldsymbol{r}^{\prime}\right) d S^{\prime}\right. \\
& -\frac{\nabla}{j \omega \varepsilon_{B}} \int_{B} G_{B}\left(\boldsymbol{r}, \boldsymbol{r}^{\prime}\right) \nabla \cdot \boldsymbol{J}\left(\boldsymbol{r}^{\prime}\right) d S^{\prime} \\
& \left.+\nabla \times \int_{S} G_{B}\left(\boldsymbol{r}, \boldsymbol{r}^{\prime}\right) \boldsymbol{M}\left(\boldsymbol{r}^{\prime}\right) d S^{\prime}\right]_{\boldsymbol{r} \in S^{-}} \\
& \boldsymbol{n} \times \boldsymbol{H}^{\mathrm{inc}}(\boldsymbol{r})=\boldsymbol{n} \times\left[j \omega \varepsilon(\boldsymbol{r}) \int_{S} \stackrel{\overrightarrow{\boldsymbol{K}}}{F}_{F}\left(\boldsymbol{r}, \boldsymbol{r}^{\prime}\right) \cdot \boldsymbol{M}\left(\boldsymbol{r}^{\prime}\right) d S^{\prime}\right. \\
& -\frac{\nabla}{j \omega \mu(\boldsymbol{r})} \int_{S} K_{\phi m}\left(\boldsymbol{r}, \boldsymbol{r}^{\prime}\right) \nabla \cdot \boldsymbol{M}\left(\boldsymbol{r}^{\prime}\right) d S^{\prime} \\
& \left.-\nabla \times \int_{S} \stackrel{\Leftrightarrow}{\boldsymbol{G}}_{A}\left(\boldsymbol{r}, \boldsymbol{r}^{\prime}\right) \cdot \boldsymbol{J}\left(\boldsymbol{r}^{\prime}\right) d S^{\prime}\right]_{\boldsymbol{r} \in S^{+}} \\
& +\boldsymbol{n} \times\left[j \omega \varepsilon_{B} \int_{S} G_{B}\left(\boldsymbol{r}, \boldsymbol{r}^{\prime}\right) M\left(\boldsymbol{r}^{\prime}\right) d S^{\prime}\right. \\
& -\frac{\nabla}{j \omega \mu_{B}} \int_{S} G_{B}\left(\boldsymbol{r}, \boldsymbol{r}^{\prime}\right) \nabla \cdot \boldsymbol{M}\left(\boldsymbol{r}^{\prime}\right) d S^{\prime} \\
& \left.-\nabla \times \int_{S} G_{B}\left(\boldsymbol{r}, \boldsymbol{r}^{\prime}\right) \boldsymbol{J}\left(\boldsymbol{r}^{\prime}\right) d S^{\prime}\right]_{\boldsymbol{r} \in S^{-}}
\end{aligned}
$$

similar to those given in [17] and [24] for PEC scatterers. These integral equations have the same form as those typically used for scattering from dielectric scatterers in free space [3]-[9], for which well established numerical procedures are available [3]-[9]. The source and observation point are described by $\boldsymbol{r}^{\prime}$ and $\boldsymbol{r}$, respectively, and $\boldsymbol{E}^{\text {inc }}(\boldsymbol{r})$ and $\boldsymbol{H}^{\text {inc }}(\boldsymbol{r})$ are the known incident electric and magnetic fields. The layered medium (complex) total permittivity and permeability are represented by $\varepsilon(\boldsymbol{r})=\varepsilon^{\prime}(\boldsymbol{r})-j \sigma(\boldsymbol{r}) / \omega$ and $\mu(\boldsymbol{r})$, respectively, where $\varepsilon^{\prime}(\boldsymbol{r})$ is the real part of the permittivity and $\sigma(\boldsymbol{r})$ is the conductivity; $\varepsilon_{B}=\varepsilon_{B}^{\prime}-j \sigma_{B} / \omega$ and $\mu_{B}$ represent these same properties for the homogeneous, lossy BOR target. Surface charge densities have been replaced by derivatives of the electric and magnetic surface current densities $\boldsymbol{J}\left(\boldsymbol{r}^{\prime}\right)$ and $\boldsymbol{M}\left(\boldsymbol{r}^{\prime}\right)$, respectively, using the continuity relation.

Explicit expressions for the spectral domain representation of the layered-medium dyadic kernel $\stackrel{\leftrightarrow}{\boldsymbol{K}}_{A}\left(\boldsymbol{r}, \boldsymbol{r}^{\prime}\right)$, the dyadic Green's function $\stackrel{\vec{G}}{A}_{A}\left(\boldsymbol{r}, \boldsymbol{r}^{\prime}\right)$ (representing the magnetic vector potential $\boldsymbol{A}(\boldsymbol{r})$ produced by an infinitesimal electric dipole at $\left.\boldsymbol{r}^{\prime}\right)$ and the electric scalar potential $K_{\phi e}\left(\boldsymbol{r}, \boldsymbol{r}^{\prime}\right)$ of a point charge associated with a horizontal electric dipole have been given by Michalski and Zheng [24], where we use their "formulation C." Generalizing the formulation in [24] to (lossy) dielectric scatterers, we introduce the additional dyadics $\stackrel{\vec{K}}{F}_{F}\left(\boldsymbol{r}, \boldsymbol{r}^{\prime}\right)$ and $\stackrel{\vec{G}}{F}_{F}\left(\boldsymbol{r}, \boldsymbol{r}^{\prime}\right)$ as well as a scalar kernel function $K_{f m}\left(\boldsymbol{r}, \boldsymbol{r}^{\prime}\right)$ associated with equivalent magnetic surface currents and surface charges, respectively; these Green's function components
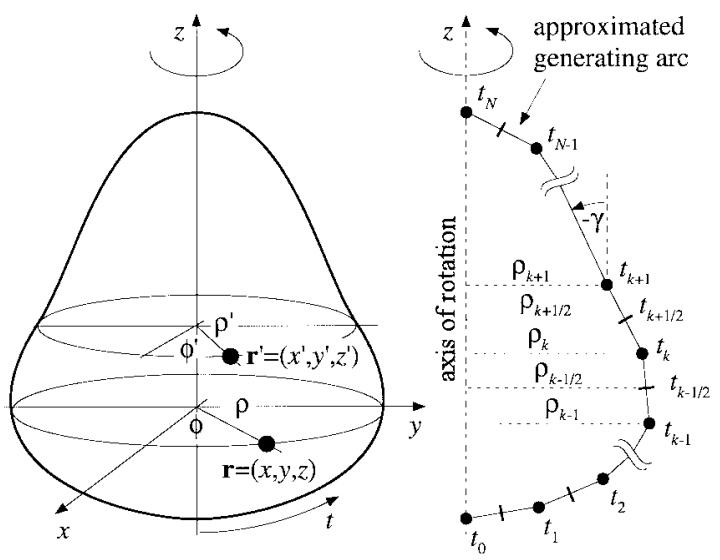

Fig. 2. Geometry for method of moments analysis of scattering from body of revolution.

can be determined from the aforementioned electric-current Green's function, via duality. For calculating the field inside the homogeneous BOR, produced by surface currents and surface charges on its surface, we utilize the homogeneousmedium Green's function $G_{B}\left(\boldsymbol{r}, \boldsymbol{r}^{\prime}\right)=\exp \left(-j k_{B} R\right) / 4 \pi R$, where $R=\left|\boldsymbol{r}-\boldsymbol{r}^{\prime}\right|$ represents the distance between source and observation point and $k_{B}$ denotes the (complex) wave number inside the BOR.

According to "formulation C" in [24], the dyadic kernel functions $\overrightarrow{\boldsymbol{K}}_{A, F}\left(\boldsymbol{r}, \boldsymbol{r}^{\prime}\right)$ and dyadic Green's functions $\stackrel{\overrightarrow{\boldsymbol{G}}}{A, F}_{\left(\boldsymbol{r}, \boldsymbol{r}^{\prime}\right) \text { are written as }}$

$$
\begin{aligned}
\dot{\boldsymbol{K}}_{A, F}\left(\boldsymbol{r}, \boldsymbol{r}^{\prime}\right)= & (\hat{\boldsymbol{x}} \hat{\boldsymbol{x}}+\hat{\boldsymbol{y}} \hat{\boldsymbol{y}}) K_{A, F}^{x x}+\hat{\boldsymbol{x}} \hat{\boldsymbol{z}} K_{A, F}^{x z}+\hat{\boldsymbol{z}} \hat{\boldsymbol{x}} K_{A, F}^{z x} \\
& +\hat{\boldsymbol{y}} \hat{\boldsymbol{z}} K_{A, F}^{y z}+\hat{\boldsymbol{z}} \hat{\boldsymbol{y}} K_{A, F}^{z y}+\hat{\boldsymbol{z}} \hat{\boldsymbol{z}} K_{A, F}^{z z} \\
\dot{\boldsymbol{G}}_{A, F}\left(\boldsymbol{r}, \boldsymbol{r}^{\prime}\right)= & (\hat{\boldsymbol{x}} \hat{\boldsymbol{x}}+\hat{\boldsymbol{y}} \hat{\boldsymbol{y}}) G_{A, F}^{x x}+\hat{\boldsymbol{z}} \hat{\boldsymbol{x}} G_{A, F}^{z x} \\
& +\hat{\boldsymbol{z}} \hat{\boldsymbol{y}} G_{A, F}^{z y}+\hat{\boldsymbol{z}} \hat{\boldsymbol{z}} G_{A, F}^{z z}
\end{aligned}
$$

The coupled integral equations in (1) are valid for an arbitrarily shaped homogeneous scatterer, for which a 3-D MoM solution could be applied. However, to make an application to ultrawide-band scattering tractable, we consider the special case of a BOR (Fig. 1), which is formed by rotating a generating arc about the $z$-axis. For numerical simulations, the generating arc is approximated by a sequence of linear segments (Fig. 2). Coordinates $(\phi, t)$ are introduced, where the angle $\phi$ is as used in cylindrical coordinates $(\rho, \phi, z)$ and $t$ is a length variable along the arc. In addition, $\gamma$ is defined as the angle between the vector $\hat{\boldsymbol{t}}$ along the arc and the $z$-direction (Fig. 2).

If the Green's function components in the spatial domain can be computed efficiently from their corresponding closedform spectral domain representation [24], the coupled system of integral equations given by (1) can be solved using a modified free-space MoM code for scattering from a BOR [3]-[9].

\section{B. Discrete Complex Image Technique}

In [17], [18], [27]-[29], [35], and [36], it was shown that the discrete complex image technique is well suited for fast 
numerical calculation of the spatial domain Green's functions, whereby laborious and computationally expensive integration of Sommerfeld type integrals is avoided. Here we summarize the basic steps.

Assuming the Green's function in the spectral domain is represented by $\tilde{f}\left(k_{x}, k_{y}, z, z^{\prime}\right)$, its spatial domain counterpart $f\left(x-x^{\prime}, y-y^{\prime}, z, z^{\prime}\right)$ is defined by the (shifted) Fourier transform

$$
\begin{aligned}
f(x- & \left.x^{\prime}, y-y^{\prime}, z, z^{\prime}\right) \\
= & \frac{1}{(2 \pi)^{2}} \int_{-\infty}^{+\infty} \int_{-\infty}^{+\infty} \tilde{f}\left(k_{x}, k_{y}, z, z^{\prime}\right) \\
& \cdot e^{-j k_{x}\left(x-x^{\prime}\right)-j k_{y}\left(y-y^{\prime}\right)} d k_{x} d k_{y} .
\end{aligned}
$$

Introducing polar coordinates $\left(k_{\rho}, \alpha\right)$ in the spectral domain and polar coordinates $(\eta, \beta)$ to represent $\left(x-x^{\prime}, y-y^{\prime}\right)$, and taking into account that $\tilde{f}\left(k_{\rho}, \alpha, z, z^{\prime}\right)$ is independent of the angle $\alpha$, the spatial domain form (3) can be written alternatively as

$$
f\left(\eta, \beta, z, z^{\prime}\right)=\frac{1}{2 \pi} \int_{0}^{\infty} \tilde{f}\left(k_{\rho}, z, z^{\prime}\right) J_{0}\left(k_{\rho} \eta\right) k_{\rho} d k_{\rho} .
$$

To avoid direct numerical integration of this highly oscillatory Sommerfeld type integral, we represent $\tilde{f}\left(k_{\rho}, z, z^{\prime}\right)$ in a form suitable for use of the Sommerfeld identity [27]-[29], [37]

$$
\frac{e^{-j k R}}{4 \pi R}=\frac{1}{2 \pi} \int_{0}^{\infty} \frac{1}{j 2 k_{z}} e^{-j k_{z} u\left(z-z^{\prime}\right)} J_{0}\left(k_{\rho} \eta\right) k_{\rho} d k_{\rho}
$$

with

$$
\begin{aligned}
k_{2} & =k_{\rho}^{2}+k_{z}^{2} \quad \text { and } \\
R & =\sqrt{\eta^{2}+u\left(z-z^{\prime}\right)^{2}} \\
& =\sqrt{\left(x-x^{\prime}\right)^{2}+\left(y-y^{\prime}\right)^{2}+u\left(z-z^{\prime}\right)^{2}} .
\end{aligned}
$$

For the source and observation point both in the $i$ th layer, this is accomplished by performing an exponential parameter fit to the spectral domain representation of the Green's function along a proper path in the $k_{z i}$-plane or $k_{\rho}$-plane [27]-[29], [36]

$$
\begin{aligned}
\tilde{f}\left(k_{\rho}, z, z^{\prime}\right)= & \frac{e^{-j k_{z i} g\left(z, z^{\prime}\right)}}{j 2 k_{z i}} \tilde{h}\left(k_{\rho}\right) \\
\approx & \frac{e^{-j k_{z i} g\left(z, z^{\prime}\right)}}{j 2 k_{z i}} \\
& \cdot\left(\lim _{k_{\rho} \rightarrow \infty} \tilde{h}\left(k_{\rho}\right)+\sum_{\mu=1}^{M} a_{\mu} e^{-k_{z i} b_{\mu}}\right) .
\end{aligned}
$$

Here we use a least-square Prony's method [30], [31] to determine the complex coefficients $a_{\mu}$ and $b_{\mu}$, although any such algorithm (e.g., the Matrix Pencil method [32], [33]) can be utilized. According to (6), we perform a parameter fit using a finite number of complex exponentials for the function $\tilde{h}\left(k_{\rho}\right)-\tilde{h}(\infty)$ (a different such for each Greens function component). Typically between $M=6$ and $M=15$ exponentials are sufficient to achieve an agreement between the approximate and the original spectral domain Green's function within $1 \%$ in the root mean square sense. For a detailed discussion, including the proper choice of the integration path, we refer the reader to [27]-[29] and [36].

\section{MoM Solution}

The coupled integral equations given by (1) are solved numerically via the well-known MoM solution for axially symmetric objects [1]-[9], [17], [18]. To take advantage of the rotational symmetry, the incident field, surface currents, and Green's functions are expanded into discrete Fourier series along the azimuthal ( $\phi$ or $\left.\phi^{\prime}\right)$ direction. The currents are represented using a subsectional pulse basis along the generating arc (coordinate $t$ ) and Fourier series expansion for the periodic azimuthal variation [5], [7]. The discretization of the generating arc and all geometrical parameters are defined in Fig. 2. Since the discrete complex image technique represents the layered-medium Green's functions as a sum of terms similar to the free-space Green's function, generalizing a freespace BOR MoM code [1]-[9] to the layered case considered here is straightforward, though somewhat laborious. Therefore, we do not repeat all the details.

Testing the integral equations corresponding to $\phi$ - and $t$ components of the incident field decouples the azimuthal Fourier modes $m=0, \pm 1, \pm 2, \pm 3, \cdots$ [1]-[9] and leads to a set of simultaneous equations where each of them may be represented in matrix form as

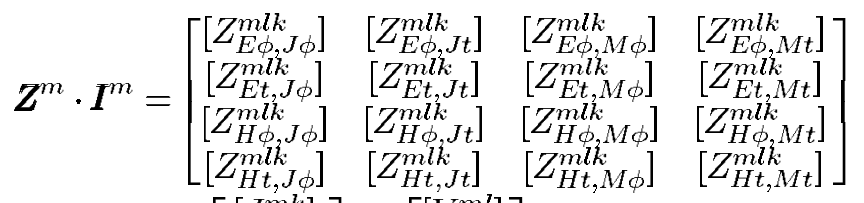

$$
\begin{aligned}
& \cdot\left[\begin{array}{c}
{\left[J_{\phi}^{m k}\right]} \\
{\left[J_{t}^{m k}\right]} \\
{\left[M_{\phi}^{m k}\right]} \\
{\left[M_{t}^{m k}\right]}
\end{array}\right]=\left[\begin{array}{l}
{\left[V_{E \phi}^{m l}\right]} \\
{\left[V_{E t}^{m l}\right]} \\
{\left[V_{H \phi}^{m l}\right]} \\
{\left[V_{H t}^{m l}\right]}
\end{array}\right]=V^{m} .
\end{aligned}
$$

$\boldsymbol{Z}^{m}$ is the moment matrix, $\boldsymbol{I}^{m}$ is a column vector containing the unknown surface current coefficients, and $V^{m}$ is the driving vector depending on the Fourier series coefficients $(m$ th mode) of the incident electric and magnetic fields tangential to the BOR.

\section{Time Domain Far-Field Plane-Wave Scattering}

The MoM impedance matrices $\boldsymbol{Z}^{m}$ are independent of the incident field (driving vector) as well as the observation point. Therefore once the impedance matrices have been calculated, the scattering for an arbitrary number of incident fields (nearor far-field source) and/or observations points (in the near or far field) can be analyzed.

Here we are only interested in the scattered field at a point $\left(r, \theta_{\text {scat }}, \psi_{\text {scat }}\right)$ in medium 1 far from the target, produced by an incident plane wave (propagating in medium 1, e.g., air) of arbitrary polarization and direction of propagation described by $\left(\theta_{\text {inc }}, \psi_{\text {inc }}\right)$, where for the case of a BOR we set $\psi_{\text {inc }}=0$ without any loss of generality, due to the rotational symmetry (Fig. 1). This is best described using the polarimetric scattering matrix $\boldsymbol{S}\left(\omega, \theta_{\text {inc }}, \theta_{\text {scat }}, \psi_{\text {scat }}\right)$ (in BSA, backscatter alignment convention) defined by

$$
\left[\begin{array}{c}
E_{\theta}^{\text {scat }} \\
E_{\psi}^{\text {scat }}
\end{array}\right]=\frac{e^{j k_{1} r}}{r}\left[\begin{array}{ll}
S_{\theta \theta} & S_{\theta \psi} \\
S_{\psi \theta} & S_{\psi \psi}
\end{array}\right] \cdot\left[\begin{array}{c}
E_{\theta}^{\text {inc }} \\
E_{\psi}^{\text {inc }}
\end{array}\right]
$$


from which the polarimetric radar cross section (RCS)

$$
\begin{aligned}
\operatorname{RCS}_{p q} & =\lim _{r \rightarrow \infty} 4 \pi r^{2}\left|\frac{E_{p}^{\text {scat }}}{E_{q}^{\text {inc }}}\right|^{2} \\
& =4 \pi\left|S_{p q}\right|^{2} \quad \text { with } \quad p, q=\theta, \psi
\end{aligned}
$$

can be easily determined. Similar to the scattering matrix $\boldsymbol{S}$, we define a normalized time domain response matrix (independent of the distance $r$ and, therefore, only characterizing the target itself)

$$
\begin{aligned}
& {\left[\begin{array}{cc}
w_{\theta \theta}(t) & w_{\theta \psi}(t) \\
w_{\psi \theta} & w_{\psi \psi}(t)
\end{array}\right]} \\
& \quad=\frac{1}{2 \pi} \int_{-\infty}^{+\infty}\left[\begin{array}{ll}
S_{\theta \theta}(\omega) \cdot P_{\theta}(\omega) & S_{\theta \psi}(\omega) \cdot P_{\psi}(\omega) \\
S_{\psi \theta}(\omega) \cdot P_{\theta}(\omega) & S_{\psi \psi}(\omega) \cdot P_{\psi}(\omega)
\end{array}\right] e^{j \omega t} d \omega
\end{aligned}
$$

which only depends on the incident pulses $p_{q}(t)$ or the corresponding spectra $P_{q}(\omega)$ in the two orthogonal incident polarizations $q=\theta$ and $q=\psi$. The scattered time domain far field can then be found at an arbitrary point $\left(r, \theta_{\text {scat }}, \psi_{\text {scat }}\right)$ as

$$
\begin{array}{r}
{\left[\begin{array}{c}
e_{\theta}^{\text {scat }}(t) \\
e_{\psi}^{\text {scat }}(t)
\end{array}\right]=\frac{1}{r}\left[\begin{array}{rr}
w_{\theta \theta}(t-r / c) & w_{\theta \psi}(t-r / c) \\
w_{\psi \theta}(t-r / c) & w_{\psi \psi}(t-r / c)
\end{array}\right] \cdot\left[\begin{array}{c}
E_{\theta 0} \\
E_{\psi 0}
\end{array}\right]} \\
\text { for }\left[\begin{array}{c}
e_{\theta}^{\text {inc }}(t) \\
e_{\psi}^{\text {inc }}(t)
\end{array}\right]=\left[\begin{array}{c}
E_{\theta 0} p_{\theta}(t) \\
E_{\psi 0} p_{\psi}(t)
\end{array}\right]
\end{array}
$$

assuming that medium 1 is nondispersive and lossless (e.g., air), where time domain variables are written as small letters and $t$ here represents time.

For a calculation of the scattering matrix $\boldsymbol{S}$ (for one incident angle) we solve (7) for two different driving vectors $V^{m}$, corresponding to two orthogonal polarizations. Explicit expressions for the elements of the driving vectors are given in the literature [1]-[9] for an incident plane wave in free-space (e.g., air). For the problem considered here, these expressions have to be modified, to take into account the direct (downward), reflected (upward) and/or transmitted (downward) incident field as well as the refraction at the interfaces, both depending on the layer $i$ in which the BOR is located. The number of azimuthal modes required in the Fourier series approximation of the incident fields, $m=-m_{\max } \cdots m_{\max }$, depends on $k_{\rho} \rho_{\max }=k_{1} \rho_{\max } \sin \theta_{\text {inc }}$, using the maximum radius $\rho_{\max }$ of the BOR. In our MoM implementation $m_{\max }$ is determined adaptively to guarantee errors less than $0.1 \%$ compared to the overall incident field. If medium 1 is lossless (e.g., $k_{1} \approx k_{0}$ ), this could be simplified by using the approximation

$$
\begin{array}{r}
m_{\max } \approx 9+1.07 k_{\rho} \rho_{\max }-\frac{16}{2+k_{\rho} \rho_{\max }} \\
\text { valid for } 0 \leq k_{\rho} \rho_{\max } \leq 100
\end{array}
$$

which was found to provide a close fit to $m_{\max }$ for the range indicated.

After having solved for the unknown current coefficients $I^{m}$ in (7), the dyadic Green's functions needed in the calculation of the scattered far field $E^{\text {scat }}$ are evaluated asymptotically using only the saddle point contribution, neglecting surface and leaky waves [37]. Explicit equations for the far field scattered by a BOR, taking into account the Fourier series expansion in the azimuth direction and therefore calculating the $\phi^{\prime}$-integral in the 2-D surface current integration analytically, can be easily derived by generalizing the solution given for free space [1]-[4].

\section{WIDE-BAND SCATTERING RESULTS}

The layered-medium BOR algorithm is applicable to many problems of interest in radar based land mine detection. For example, one can account for the layered manner in which soil is often distributed. Moreover, the overturned soil in which a mine is buried often has electrical properties different from those of the surrounding, undisturbed soil; the disturbed soil can be modeled approximately via a lossy dielectric BOR, in which a separate BOR (representing the model mine) is placed. Such applications are the subjects of future papers. Here we demonstrate a few examples, which show the potential and utility of the algorithm. In particular, we first examine scattering from a plastic BOR (model plastic mine) buried in a lossy, dispersive half-space (Section III-A), with half-space electrical parameters representative of data measured from real soil [38]. As a comparison, we also consider scattering from a buried perfectly conducting BOR of the same shape as the model plastic mine, with the perfectly conducting BOR modeled by use of a subset of the terms in (7) [17]. A comparison between the weak energy scattered from dielectric mines, relative to conducting mines of the same shape, underscores the difficulty of radar-based plastic mine detection. Finally, to demonstrate an example for which the layered-medium Green's function is applicable, we consider scattering from a plastic BOR buried just under the soil, with the soil covered by a layer of snow (Section III-B). In this context we examine the scattered signal as a function of snow type (snow wetness) and snow depth.

In the context of radar-based detection of buried targets, there is always a tradeoff between resolution and signal strength. To achieve good penetration in typical lossy soils, ground-penetrating radar systems are typically designed to operate at frequencies less than approximately $1.5 \mathrm{GHz}$ [11], [14], [19], [20]. However, such a restriction in bandwidth reduces resolution, thereby minimizing the information content in the scattered fields. To enhance the information in the scattered fields, one must increase the system bandwidth (resolution), although this implies increased signal attenuation in lossy soils. This issue is examined by considering several incident waveforms of differing bandwidth and center frequency.

Finally, before proceeding to the results, we note that there is very little work we are aware of published on short-pulse scattering from buried dielectric targets. The computer code was checked extensively to verify its accuracy, and tests were successfully performed for a dielectric sphere in free-space and with the limited data available on short-pulse scattering from buried targets [17].

\section{A. Dielectric and Conducting BOR Buried in a Half Space}

In our first set of examples, we consider perfectly conducting (PEC) and dielectric cylinders of the same shape, buried in 


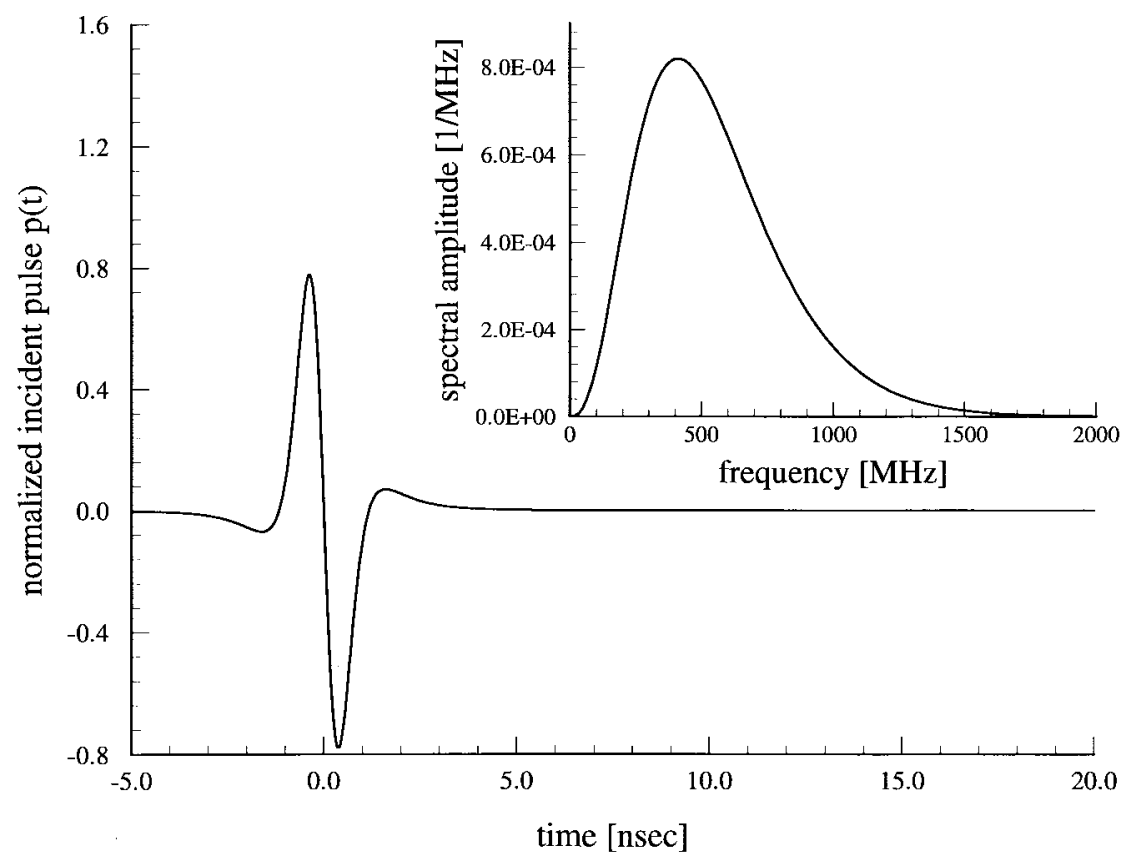

Fig. 3. Incident pulse $p(t)$ (Rayleigh pulse of order $n=3$, center frequency $f_{c}=410 \mathrm{MHz}$ [39]) and corresponding amplitude spectrum $|P(f)|$ (used in Fig. 4).

a lossy dispersive half space. The PEC and plastic targets are both cylinders of diameter $12.6 \mathrm{~cm}$ and height $5.3 \mathrm{~cm}$, with the dielectric target characterized by $\varepsilon_{r B}^{\prime}=2.9$ and $\sigma_{B}=10^{-3}$ $\mathrm{S} / \mathrm{m}$ (PVC). The top of the cylinders are placed $5 \mathrm{~cm}$ beneath the air-ground interface, and the pulsed plane wave is incident at $\theta_{\text {inc }}=50 \mathrm{deg}$ (see Fig. 1). Finally, the half-space in which these targets are buried is described by the characteristics of Puerto Rico clay (10\% water content), as reported in [38].

The first set of examples utilize the incident pulse in Fig. 3 (a Raleigh wavelet [39] of order $n=3$ and center frequency at $f_{c}=410 \mathrm{MHz}$ ). This incident waveform and bandwidth are typical of many ground-penetrating systems [11], [14], [19], [20]. The far-zone backscattered fields are normalized as in (10)-(11), with results presented in Fig. 4. For a BOR target there are no cross-polarized backscattered fields, and therefore results are only plotted for $V V$ (vertical incidence, vertical receive) and $H H$ (horizontal incidence, horizontal receive) scattering. While there are slight differences in the $V V$ and $\mathrm{HH}$ scattered fields for a given target, the most dramatic characteristic of the results in Fig. 4 is the large discrepancy in the amplitudes of the waveforms scattered from the PEC and PVC targets (despite the fact that the targets have exactly the same shape) and the different polarity of the signals, the latter resulting from a "negative" contrast in permittivity $\left(\varepsilon_{r B}^{\prime}=2.9\right.$ for the PVC target compared to a soil permittivity ranging from 5 to 6 ).

The relatively weak backscattered fields for the plastic target are attributed to the modest contrast between the target and the background soil. Over the bandwidth considered here, the Puerto Rico clay [38] has a dielectric constant ranging from 5 to 6 , as compared to $\varepsilon_{r B}^{\prime}=2.9$ for the target. It should be pointed out, however, that many dry soils have dielectric constants even closer to $\varepsilon_{r B}^{\prime}$ for which the fields scattered from the plastic target will be even smaller.

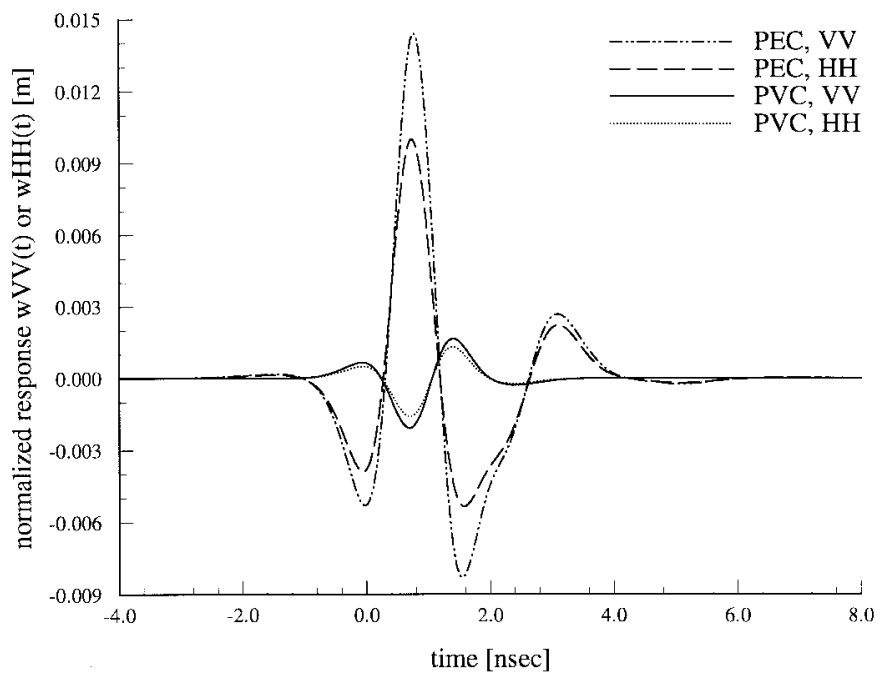

Fig. 4. Comparison of the normalized time domain response for a perfectly conducting (PEC) and a plastic (PVC: $\varepsilon_{r B}^{\prime}=2.9, \sigma_{B}=10^{-3} \mathrm{~S} / \mathrm{m}$ ) cylinder of same dimensions (diameter $=12.6 \mathrm{~cm}$, height $=5.3 \mathrm{~cm}$ ). The top of the cylinder is $5 \mathrm{~cm}$ below the ground-air interface at $z_{1}=0 \mathrm{~cm}$ (layer 1: air, layer 2: Puerto Rico clay with $10 \%$ water [38]). Results are shown for $w_{V V}(t)=w_{\theta \theta}(t)$ and $w_{H H}(t)=w_{\psi \psi}(t)$ in case of backscattering and $\theta_{\text {inc }}=50^{\circ}$ (no cross-polarization for backscattering from BOR [22]). The incident pulse is given in Fig. 3.

Another characteristic of the results in Fig. 4 is the fact that the time-domain scattered fields have duration similar to the incident waveform in Fig. 3 (although the wave shapes are distorted due to dispersive target scattering and propagation through the dispersive soil). Therefore, for the bandwidth in Fig. 3, the targets (both PEC and PVC) are dispersive point scatterers. Since there are many obstacles which will similarly be point scatterers (rocks, roots, etc.), target discrimination is difficult with this incident waveform. To address this issue, one can utilize a wider bandwidth incident waveform such 


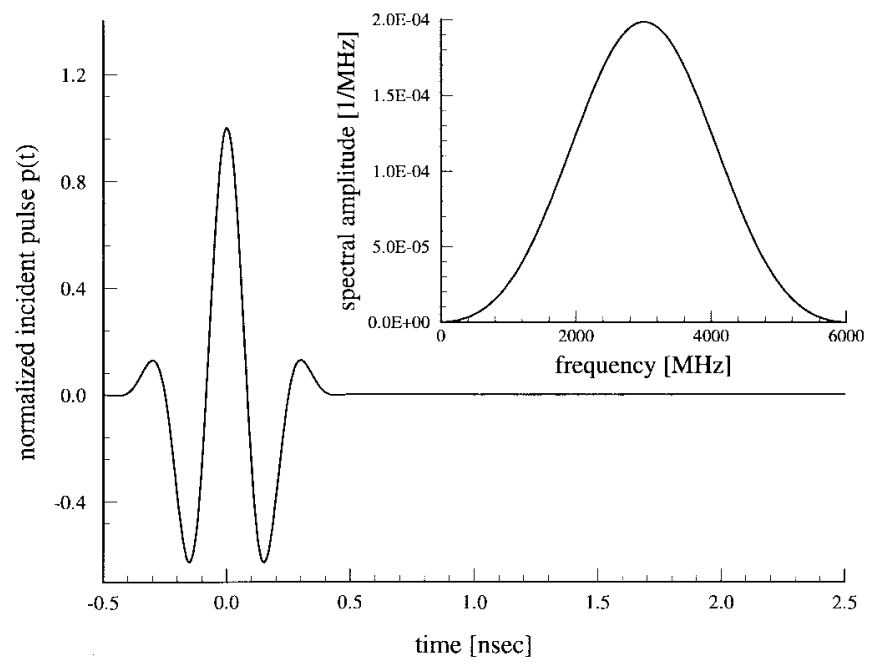

Fig. 5. Incident pulse $p(t)$ (center frequency $f_{c}=3000 \mathrm{MHz}$ ) and corresponding amplitude spectrum $|P(f)|$ (used in Fig. 6).

that higher resolution is achieved. We therefore consider the waveform in Fig. 5, which has a center frequency at $f_{c}=3000 \mathrm{MHz}$ and considerably wider bandwidth than Fig. 3 . To accommodate the increased bandwidth, however, the principal energy components are at significantly higher frequency than those in Fig. 3, resulting in increased attenuation as the wave penetrates the soil. In Fig. 6 we consider the same targets as in Fig. 4, but using the incident waveform in Fig. 5. As seen from Fig. 6(a) and (b), the increased bandwidth results in a noticeable difference in the $V V$ and $H H$ backscattered fields. Moreover, the shapes of the waveforms scattered from the PEC and PVC targets are different as well (note the different polarity already mentioned above), although, as in Fig. 4, the scattered fields from the PEC target are markedly stronger than those of the plastic target.

The increased bandwidth of Fig. 6 has resulted in the anticipated enhanced information content in the scattered fields (relative to Fig. 4). For the PEC target we attributed much of the additional waveforms (vis-à-vis, Fig. 4) to reverberations between the top of the target and the air-ground interface, which can be resolved using this larger bandwidth. For the plastic target there are also such reverberations, as well as energy that gets absorbed in and then reradiated from the interior of the plastic (these two phenomena are difficult to separate). The increased complexity in the scattered waveforms of Fig. 6 is accompanied by a significant reduction in the amplitude of the scattered fields (due to the aforementioned enhanced soil attenuation); comparing Fig. 6(a) and (b) with Fig. 4, we note that the higher bandwidth excitation results in an order-of-magnitude reduction in the scattered-field amplitudes.

\section{B. Dielectric BOR Buried Under a Snow Layer}

We consider a PVC cylinder of the same shape as above, buried $1 \mathrm{~cm}$ under soil, with the soil covered by a $10-\mathrm{cm}$ snow layer. The winter conditions are likely to alter the soil properties (due to freezing), but for simplicity, we consider the soil as in Figs. 4 and 6. Dry snow is often easily penetrated

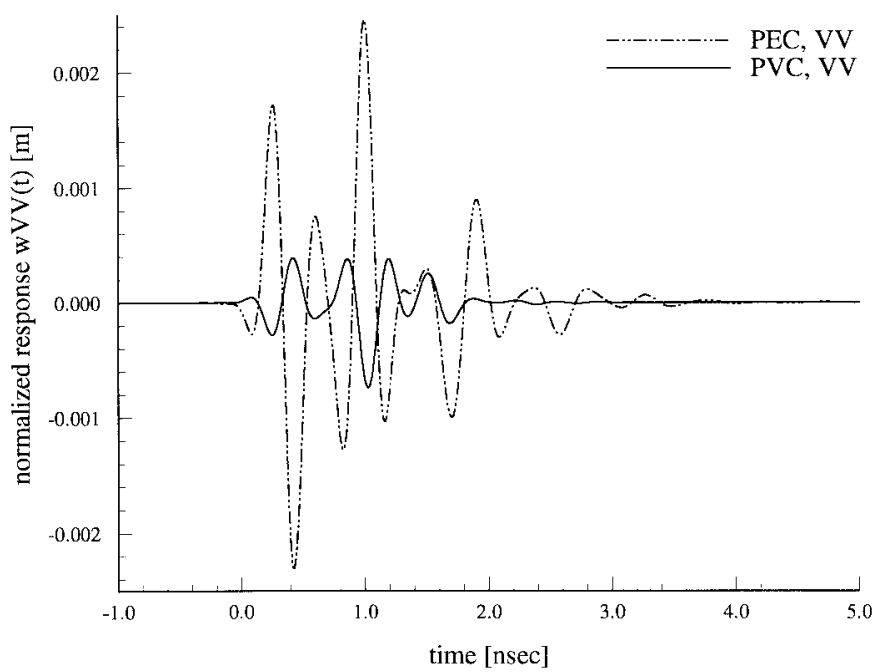

(a)

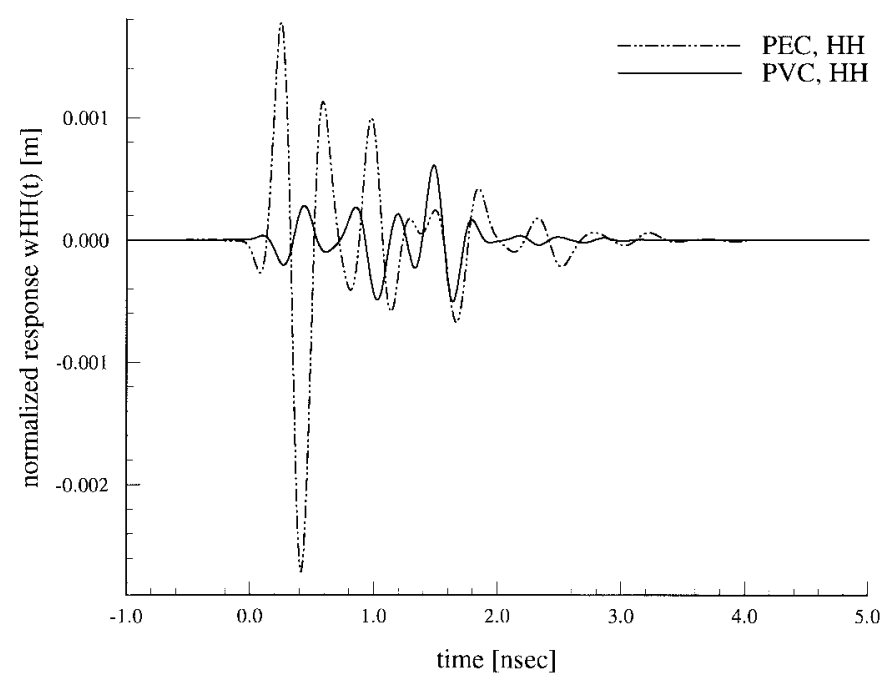

(b)

Fig. 6. Same as Fig. 4, but using pulse in Fig. 5. (a) $w_{V V}(t)=w_{\theta \theta}(t)$, (b) $w_{H H}(t)=w_{\psi \psi}(t)$.

(relative to soil) and the shallow target necessitates minimal soil penetration. Therefore, we consider the incident waveform in Fig. 7 (center frequency $f_{c}=720 \mathrm{MHz}$ ), characterized by increased bandwidth relative to the pulse in Fig. 3. In Fig. 8 we consider "dry snow," "snow," and "wet snow," characterized, respectively, by $\varepsilon_{r 2}^{\prime}=1.5$ and $\sigma_{2}=2 \cdot 10^{-4} \mathrm{~S} / \mathrm{m} ; \varepsilon_{r 2}^{\prime}=3$ and $\sigma_{2}=10^{-3} \mathrm{~S} / \mathrm{m} ; \varepsilon_{r 2}^{\prime}=5$ and $\sigma_{2}=10^{-2} \mathrm{~S} / \mathrm{m}$ [40]. For the $V V$ results in Fig. 8(a), the increase in dielectric constant with snow wetness results in an expected temporal shift, but the general shape of the scattered waveform is largely unchanged with snow type. As a comparison, in Fig. 8(b), we consider $\mathrm{HH}$ scattering for which the scattered fields are characterized by a more substantial variation with snow type. Interestingly, in Fig. 8(b), the 10-cm layer of "dry snow" results in a stronger scattered field thatn when there is no snow at all; this is attributed to the very low loss of the dry snow and a snow-layer-induced enhanced impedance matching between the soil and air. The difference between the $V V$ and $H H$ cases is demonstrated even more dramatically in Fig. 9 for 


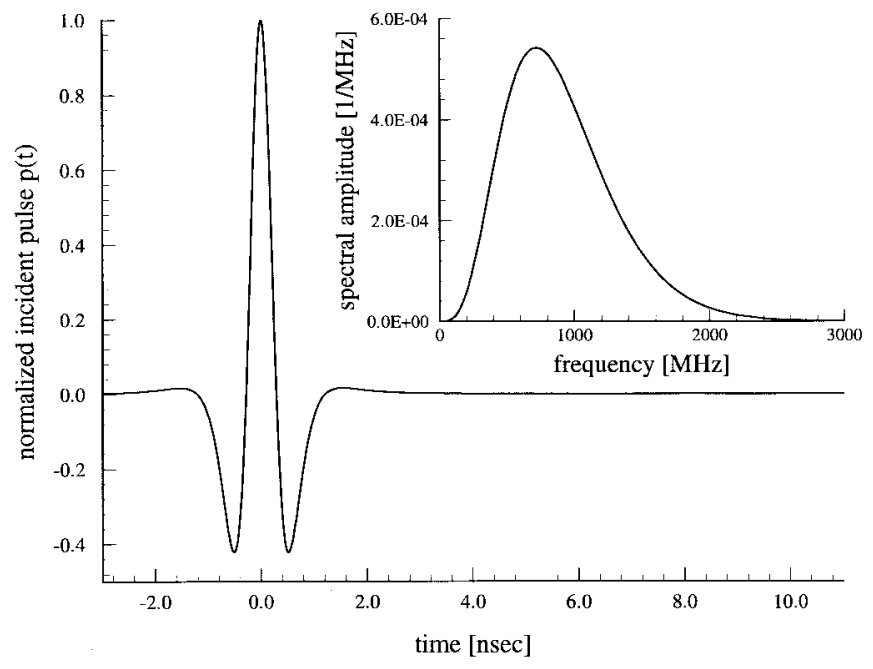

Fig. 7. Incident pulse $p(t)$ (Rayleigh pulse of order $n=4$, center frequency $f_{c}=720 \mathrm{MHz}[39]$ ) and corresponding amplitude spectrum $|P(f)|$ (used in Figs. 8 and 9).

which the backscattered fields are examined as a function of snow depth $(0,5,10,15,20 \mathrm{~cm})$ for the "snow" of Fig. 8. For $V V$ polarization there is verly little reverberation in the snow layer, while for $H H$ polarization, there is a noticeable late-time oscillation manifested with increased snow depth. The minimal $V V$ reverberation is attributed to the fact that there is near-total transmission at the various interfaces (particularly at the air-snow interface), due to near-Brewsterangle excitation [41], while such Brewster effects are absent for the $H H$ case.

\section{CONCLUSIONS}

A numerical algorithm has been developed for modeling plastic mines buried in a lossy layered medium (e.g., soil with or without a snow layer). To make such an analysis tractable for the wide-band, short-pulse problems of interest, we have assumed that the mine can be modeled as a BOR, which is a good approximation for most mines. Exploitation of this feature results in a significant savings in required computational resources (vis-à-vis finite elements [23] or the finite difference time domain [20]). While the MoM BOR algorithm has been utilized for some time, it is only through application of the method of complex images [27]-[29], for efficient evaluation of the layered-medium Green's function, that the wide-band computations of interest here are tractable. The wide-band scattering results for the PEC and PVC BOR's presented above took typically on the order of one and four hours of CPU time, respectively, on a 200-MHz Pentium Pro personal computer (with the code written in Fortran 77).

As was demonstrated in the results of Section III, the characteristics of the scattered waveform depend strongly on the environment, mine type, and system bandwidth. To deploy a radar system properly for a given application, it is important that the expected scattered signal amplitude and shape be known in advance (there are many plastic mines and soil types for which radar-based detection is not

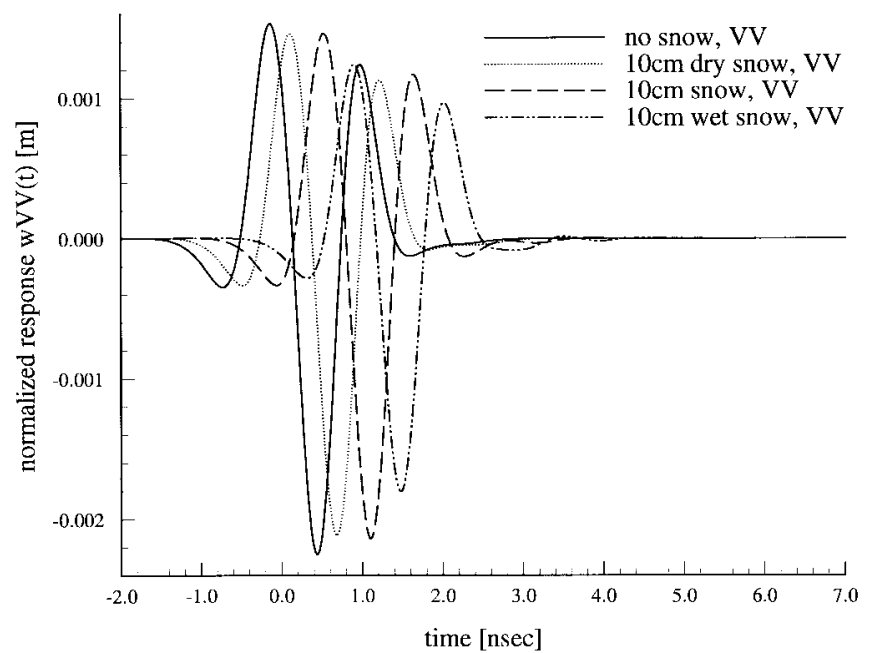

(a)

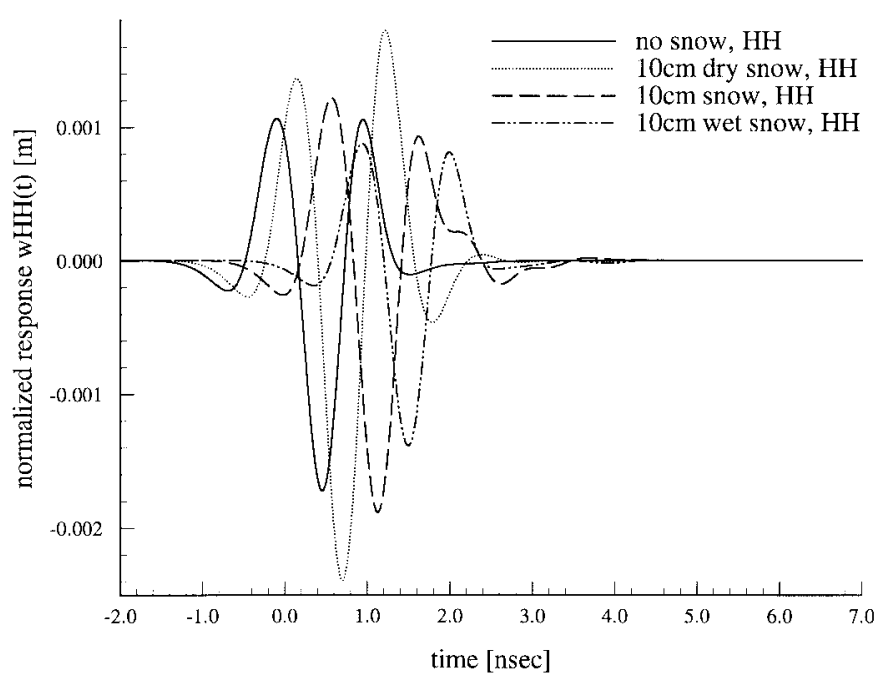

(a)

Fig. 8. Normalized time domain response for a buried PVC-cylinder (diameter $=12.6 \mathrm{~cm}$, height $=5.3 \mathrm{~cm}, \varepsilon_{r B}^{\prime}=2.9, \sigma_{B}=10^{-3} \mathrm{~S} / \mathrm{m}$ ) including the effect of a 10-cm snow layer (layer 2: "dry snow" with $\varepsilon_{r 2}^{\prime}=1.5$ and $\sigma_{2}=2 \cdot 10^{-4} \mathrm{~S} / \mathrm{m}$; "snow" with $\varepsilon_{r 2}^{\prime}=3$ and $\sigma_{2}=10^{-3} \mathrm{~S} / \mathrm{m}$; "wet snow" with $\varepsilon_{r 2}^{\prime}=5$ and $\sigma_{2}=10^{-2} \mathrm{~S} / \mathrm{m}$ ) on top of the soil (layer 3: Puerto Rico clay with $10 \%$ water content [38]). The top of the cylinder is $1 \mathrm{~cm}$ below the ground-snow interface at $z_{2}=0 \mathrm{~cm}$, the snow-air interface is located at $z_{1}=10 \mathrm{~cm}$. Results are shown for backscattering and $\theta_{\text {inc }}=60^{\circ}$. The incident pulse is given in Fig. 7. (a) $w_{V V}(t)=w_{\theta \theta}(t)$. (b) $w_{H H}(t)=w_{\psi \psi}(t)$.

a viable option, especially when the contrast between the dielectric target and soil is small). It is for such purposes that the numerical analysis presented here is of particular importance. Moreover, in the design of optimal detectors [42], one requires a priori knowledge of the target signature as a function of soil type and target depth (the latter two are not known exactly in practice and, therefore, must be characterized statistically). For such applications, one must have access to the target signature for a large range of environmental conditions, necessitating the efficient algorithm presented here.

While the layered-medium BOR algorithm is applicable to many problems of interest in mine detection, here we have chosen to present a few representative examples. As expected, 


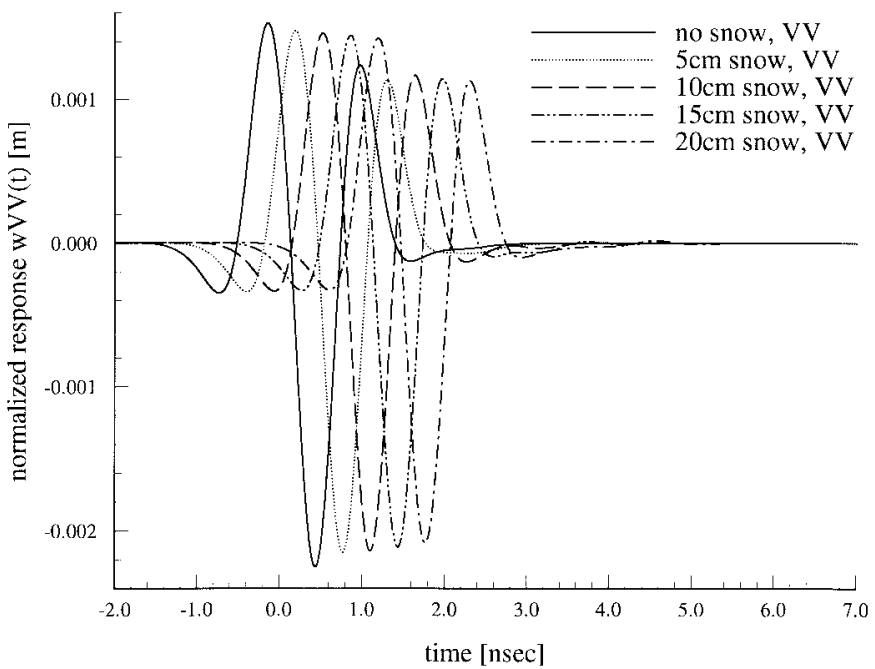

(a)

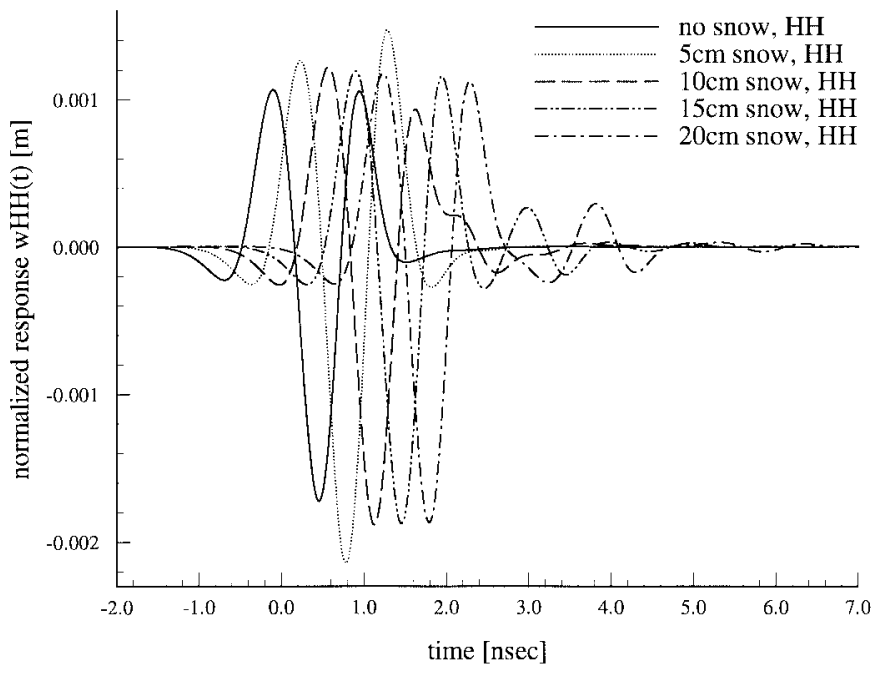

Member, IEEE

(b)

Fig. 9. Same as in Fig. 8, but considering variable snow depths $\left(z_{1}=0,5,10,15,20 \mathrm{~cm}\right)$ and "snow" with $\varepsilon_{r 2}^{\prime}=3$ and $\sigma_{2}=10^{-3} \mathrm{~S} / \mathrm{m}$. (a) $w_{V V}(t)=w_{\theta \theta}(t)$. (b) $w_{H H}(t)=w_{\psi \psi}(t)$.

plastic targets result in significantly reduced scattered energy (relative to a conducting target of the same shape), with the details of the plastic-target signature dependent on the contrast between the electrical properties of the soil and target. We also examined scattering from targets buried under a snow layer. For dry snow, the attenuation introduced by snow penetration is minimal at the frequencies of interest, and radar provides an effective tool for subsurface sensing. We also witnessed difference in the $V V$ and $H H$ scattered fields in such environments due primarily to Brewster-angle effects.

An advantage of the frequency-domain solution presented here (relative to time-domain techniques, such as the finite difference time domain [20]) is its applicability to the computation of target resonances [18], [21]. The late-time resonances of buried plastic targets have been measured previously, with particularly encouraging results presented in [14]. However, as for the scattering problem addressed here, the details of the buried-plastic-target resonances are strongly dependent on the specifics of the target and background. To quantify the regimes for which resonance-based discrimination is appropriate, it is important to have an accurate model. The MoM analysis of buried-plastic-target (mine) resonances will be the subject of future research.

\section{REFERENCES}

[1] M. G. Andreasen, "Scattering from bodies of revolution," IEEE Trans. Antennas Propagat., vol. 13, pp. 303-310, Mar. 1965.

[2] J. R. Mautz and R. F. Harrington, "Radiation and scattering from bodies of revolution," Appl. Sci. Res., vol. 20, pp. 405-435, June 1969.

[3] T. Wu and L. L. Tsai, "Scattering from arbitrarily-shaped lossy dielectric bodies of revolution," Radio Sci., vol. 12, pp. 709-718, Oct. 1977.

[4] J. R. Mautz and R. F. Harrington, "Electromagnetic scattering from a homogeneous material body of revolution," $A E \ddot{U}$, vol. 33, pp. 71-80, Feb. 1979.

[5] A. W. Glisson and D. R. Wilton, "Simple and efficient numerical methods for problems of electromagnetic radiation and scattering from surfaces," IEEE Trans. Antenans Propagat., vol. 28, pp. 593-603, Sept. 1980.

[6] A. W. Glisson, D. Kajfez, and J. James, "Evaluation of modes in dielectric resonators using a surface integral equation formulation," IEEE Trans. Microwave Theory Tech., vol. 31, pp. 1023-1029, Dec. 1983.

[7] A. W. Glisson, "Integral equation techniques," in Dielectric Resonators, D. Kajfez and P. Guillon, Eds. Dedham, MA: Artech House, 1986, pp. 259-325.

[8] D. Kajfez, A. W. Glisson, and J. James, "Computed modal field distributions for isolated dielectric resonators," IEEE Trans. Micowave Theory Tech., vol. 32, pp. 1609-1616, Dec. 1984

[9] S. D. Gedney and R. Mittra, "The use of the FFT for the efficient solution of the problem of electromagnetic scattering by a body of revolution," IEEE Trans. Antennas Propagat., vol. 38, pp. 313-322, Mar. 1990.

[10] R. W. P. King and C. W. Harrison, "The transmission of electromagnetic waves and pulses into the earth," J. Appl. Phys., vol. 39, pp. 4444-4452, Aug. 1968.

[11] D. L. Moffatt and R. J. Puskar, "A subsurface electromagnetic pulse radar," Geophys., vol. 41, pp. 506-518, June 1976.

[12] G. S. Smith and W. R. Scott, "A scale model for studying ground penetrating radars," IEEE Trans. Geosci. Remote Sensing, vol. 27, pp. 358-363, July 1989.

[13] C. Liu and L. C. Shen, "Numerical simulation of subsurface radar for detecting buried pipes," IEEE Trans. Geosci. Remote Sensing, vol. 29, pp. 795-798, Sept. 1991.

[14] L. Peters, Jr., J. J. Daniels, and J. D. Young, "Ground penetrating radar as a subsurface environmental sensing tool," Proc. IEEE, vol. 82, pp. 1802-1822, Dec. 1994.

[15] A. C. Dubey and R. L. Barnard, Eds., "Detection and remediation technologies for mines and minelike targets," SPIE Proc., Orlando, FL, vol. 3079, 1997.

[16] S. Vitebskiy and L. Carin, "Moment-method modeling of short-pulse scattering from and the resonances of a wire buried inside a lossy, dispersive half-space," IEEE Trans. Antennas Propagat., vol. 43, pp. 1303-1312, Nov. 1995.

[17] S. Vitebskiy, K. Sturgess, and L. Carin, "Short-pulse plane-wave scattering from buried perfectly conducting bodies of revolution," IEEE Trans. Antennas Propagat., vol. 44, pp. 143-151, Feb. 1996.

[18] S. Vitebskiy and L. Carin, "Resonances of perfectly conducting wires and bodies of revolution buried in a lossy dispersive half-space," IEEE Trans. Antennas Propagat., vol. 44, pp. 1575-1583, Dec. 1996.

[19] S. Vitebskiy, L. Carin, M. A. Ressler, and F. H. Le, "Ultra-wide-band, short-pulse ground-penetrating radar: Simulation and measurement,' IEEE Trans. Geosci. Remote Sensing, vol. 35, pp. 762-772, May 1997.

[20] J. M. Bourgeois and G. S. Smith, "A fully three-dimensional simulation of a ground-penetrating radar: FDTD theory compared with experiment," IEEE Trans. Geosci. Remote Sensing, vol. 34, pp. 36-44, Jan. 1996.

[21] N. Geng, D. Jackson, and L. Carin, "On the resonances of a dielectric BOR buried in a dispersive layered medium," submitted to IEEE Trans. Antennas Propagat.

[22] L. Carin, R. Kapoor, and C. E. Baum, "Polarimetric SAR imaging of buried landmines," IEEE Trans. Geosci. Remote Sensing, vol. 36, pp. 1985-1988, Nov. 1998. 
[23] H. S. Chang and K. K. Mei, "Scattering of electromagnetic waves by buried and partly buried bodies of revolution," IEEE Trans. Geosci. Remote Sensing, vol. 23, pp. 596-605, 1985.

[24] K. A. Michalski and D. Zheng, "Electromagnetic scattering and radiation by surfaces of arbitrary shape in layered media, Part I: Theory," IEEE Trans. Antennas Propagat., vol. 38, pp. 335-344, Mar. 1990.

[25] J. R. Wait, "Image theory of a quasistatic magnetic dipole over a dissipative half-space," Electron. Lett., vol. 5, pp. 281-282, May 1969.

[26] I. V. Lindell and E. Alanen, "Exact image theory for the Sommerfeld half-space problem, Part III: General formulation," IEEE Trans. Antennas Propagat., vol. 32, pp. 1027-1032, Oct. 1984.

[27] Y. L. Chow, J. J. Yang, D. G. Fang, and G. E. Howard, "A closed-form spatial Green's function for the thick microstrip substrate," IEEE Trans. Microwave Theory Tech., vol. 39, pp. 588-592, Mar. 1991.

[28] J. J. Yang, Y. L. Chow, and D. G. Fang, "Discrete complex images of a three-dimensional dipole above and within a lossy ground," Proc. Inst. Elect. Eng., vol. 138, pt. H, pp. 319-326, Aug. 1991.

[29] R. M. Shubair and Y. L. Chow, "A simple and accurate complex image interpretation of vertical antennas present in contiguous dielectric halfspaces," IEEE Trans. Antennas Propagat., vol. 41, pp. 806-812, June 1993.

[30] A. J. Poggio, M. L. Van Blaricum, E. K. Miller, and R. Mittra, "Evaluation of a processing technique for transient data," IEEE Trans. Antennas Propagat., vol. 26, pp. 165-173, Jan. 1978.

[31] M. L. Van Blaricum and R. Mittra, "A technique for extracting the poles and residues of a system directly from its transient response," IEEE Trans. Antennas Propagat., vol. 23, pp. 777-781, Nov. 1975.

[32] Y. Hua and T. K. Sarkar, "Generalized pencil-of-function method for extracting poles of an EM system from its transient response," IEEE Trans. Antennas. Propagat., vol. 37, pp. 229-234, Feb. 1989.

[33] _ "Matrix pencil method for estimating parameters of exponentially damped/undamped sinusoids in noise," IEEE Trans. Acoust., Speech, Signal Processing, vol. 38, pp. 814-824, May 1990.

[34] Y. Rahmat-Samii, R. Mittra, and P. Parhami, "Evaluation of Sommerfeld integrals for lossy half-space problems," Electromagn., vol. 1, no. 1, pp. $1-28,1981$.

[35] A. K. Abdelmageed and K. A. Michalski, "Analysis of EM scattering by conducting bodies of revolution in layered media using the discrete complex image method," in IEEE Antennas Propagat. Symp. Dig., Newport Beach, CA, June 1995.

[36] M. I. Aksun, "A robust approach for the derivation of closed-form Green's functions," Trans. Microwave Theory Tech., vol. 44, pp. 651-658, May 1996.

[37] L. B. Felsen and N. Marcuvitz, Radiation and Scattering of Waves. Englewood Cliffs, NJ: Prentice-Hall, 1973.
[38] J. E. Hipp, "Soil electromagnetic parameters as functions of frequency, soil density, and soil moisture," Proc. IEEE, vol. 62, pp. 98-103, Jan. 1974.

[39] P. Hubral and M. Tygel, "Analysis of the Rayleigh pulse," Geophysics, vol. 54, pp. 654-658, May 1989.

[40] F. T. Ulaby, R. K. Moore, and A. K. Fung, Microwave Remote Sensing. Active and Passive, vol. I. Reading, MA: Addison-Wesley, 1982

[41] C. A. Balanis, Advanced Engineering Electromagnetics. New York: Wiley, 1989.

[42] L. Carin, N. Geng, M. McLure, J. Sichina, and L. Nguyen, "Ultra-wideband synthetic-aperture radar for mine-field detection," IEEE Antennas Propagat. Mag., vol. 41, pp. 18-33, Feb. 1999.

Norbert Geng (S'91-M'96) was born on May 14, 1965, in Lauchringen, Germany. He received the Dipl.-Ing. and Dr.-Ing. degrees in electrical engineering from the University of Karlsruhe, Germany, in 1991 and 1996, respectively.

From 1991 to 1996, he was with the Institute for Microwaves and Electronics at the University of Karlsruhe, working on full-wave propagation modeling for radio communication systems. He received the Mannesmann Innovation Award in 1997 for his Ph.D. thesis on this subject. In January 1997 he joined the Department of Electrical and Computer Engineering at Duke University, Durham, NC, as a Visiting Post-Doctoral Fellow for 18 months. Since July 1998 he has been back with the University of Karlsruhe. His current research interests include computational methods in electromagnetics and wave propagation modeling.

Lawrence Carin (SM'96) was born on March 25, 1963 in Washington, DC. He received the B.S., M.S., and Ph.D. degrees in electrical engineering from the University of Maryland, College Park, in 1985, 1986, and 1989, respectively.

In 1989, he joined the Electrical Engineering Department at Polytechnic University, Brooklyn, as an Assistant Professor, and became an Associate professor there in 1994. In September 1995, he joined the Electrical Engineering Department at Duke University, where he is an Associate Professor. His current research interests include short-pulse scattering, propagation and signal processing. He is the principal investigator on an Army Research Office funded Multidisciplinary University Research Initiative (MURI) on demining. Dr. Carin is a member of the Tau Beta Pi and Eta Kappa Nu honor societies. 